\title{
IL PROBLEMA DELL'ETERNITÀ DEL MONDO NEL PENSIERO DI MOSÈ MAIMONIDE
}

ERMENEGILDO BERTOLA

Università Cattolica di Milano

La posizione che il Maimonide assunse circa il problema dell'eternità o meno del mondo è unica nella storia del pensiero giudaico e musulmano; su questo punto il Maimonide ebbe, se così si può dire, un solo seguace, Tommaso d'Aquino, il più grande dei pensatori cristiani del medioevo, anche se la posizione di Tommaso non coincide totalmente con quella del Maimonide ${ }^{\text {. }}$

Per comprendere esattamente la posizione del Maimonide, occorre tenere presenti due aspetti della sua esposizione, uno è di carattere metodologico, l'altro è di carattere contenutistico. Il Maimonide distingue tra una tesi o l'affermazione di una tesi e la dimostrazione razionale della tesi stessa; il chè vuol dire che il Maimonide distingue tra l'affermazione della eternità del mondo e la prova razionale di questa eternità, tra l'affermazione della sua non eternità e la prova razionale della sua non eternità. Questo per quanto riguarda il metodo; per quanto riguarda invece il contenuto, il Maimonide lega, con particolari vincoli, il problema della eternità o meno del mondo con il problema della creazione volontaria o meno del mondo. Per lui, a differenza, ad esempio, di Tommaso d'Aquino, ciò che è creato, perciò stesso che è creato volontariamente non è eterno; ed al contrario ciò che è eterno, per il fatto stesso che è eterno non è stato prodotto volontariamente, bensì necessariamente. La possibilità di una creazione volontaria $a b$ aeterno non è presa in considerazione dal Maimonide, poichè per lui, essa sarebbe una affermazione con-

\footnotetext{
' Sul problema dell'eternità del mondo in Tommaso d'Aquino, anche in relazione alla posizione del Maimonide, vedi: E. BERTOLA, "Tommaso d'Aquino e il problema dell'eternità del mondo", Rivista di Filosofia neo-scolastica 66 (1974) 312-355.
} 
traddittoria. Questo vuol dire che una dimostrazione razionale posto che tale dimostrazione esista, della creazione volontaria del mondo porta con sè, come conseguenza, la dimostrazione che il mondo non è eterno. Anche se non lo dice esplicitamente vale anche il contrario: una dimostrazione razionale, posto che esista, della eternità del mondo trascina con sè la dimostrazione che il mondo non è stato creato, o almeno, perchè su questo punto occorre particolare cautela, essa trascina con sè la dimostrazione che il mondo non è stato creato secondo il desiderio e la volontà di Dio.

Notiamo come, in conseguenza del legame che per il Maimonide sussiste tra creazione e non eternità e quindi della contraddizione che sussiste tra eternità e creazione volontaria, il Maimonide usa il termine di hiddû́s, 'novità', o forse meglio ex novo; per il Maimonide il mondo o è eterno o è ex novo, cioè creato.

Il fatto che se il mondo fosse eterno esso non sarebbe creato volontariamente, fa si che il problema della eternità o meno del mondo assuma per il Maimonide, una importanza particolare. Questo spiega perchè egli alla dottrina della creazione, secondo la rivelazione mosaica, aggiunga quella della non eternità, e sostenga che essa è il fondamento della Legge di Mosè e che essa è altresì: «il secondo fondamento rispetto all'unità [di Dio]».

Lasciamo per ora in sospeso questa seconda affermazione per sottolineare questo principio: chi nega che il mondo sia stato creato, cioè chi nega che esso non sia eterno, nega il fondamento stesso della religione ebraica, fondamento della Torah rivelata da Dio a Mosè.

Ma il Maimonide non lega soltanto, come abbiamo visto, il problema dell'eternità o meno del mondo con quello della creazione, egli lega lo stesso problema con la dottrina o con il problema del tempo. Problema che egli tratta in modo particolare proprio in questa occasione. L'esposizione del pensiero del Maimonide su questa questione dell'eternità $o$ non eternità del mondo presenta perciò qualche difficoltà per la sua complessità. Per cercare di semplificare il nostro compito e far comprendere più chiaramente il pensiero del Maimonide, risolviamo preliminarmente qui il problema del tempo.

La dottrina del Maimonide circa il tempo ha nella Guida, una trattazione sintetica, ma sufficientemente esauriente. Essa si fonda 
su questi punti: 1) il tempo ha una sua realtà, anche se esso non è una sostanza, bensì un accidente ${ }^{2}$; 2) vi sono due categorie di accidenti, gli accidenti che hanno un vincolo diretto con la sostanza di cui sono accidenti e vi sono accidenti che con la sostanza hanno soltanto un vincolo indiretto; i primi sono accidenti della sostanza i secondi sono accidenti di accidenti della propria sostanza ${ }^{3 ;}$ 3) gli accidenti non hanno una realtà indipendente, ma esistono soltanto in quanto vi è un substratum o un soggetto su cui inerire, sia questo substratum una sostanza o un altro accidente. Ora il tempo appartiene alla categoria degli accidenti di accidenti, esso è un accidente del moto, il quale moto è a sua volta un accidente ${ }^{4}$. Il fatto di essere un accidente di un accidente che fa da substratum al tempo, e che questo substratum sia qualcosa di instabile, quale è il moto, spiega perchè la nozione del tempo non sia facilmente afferrabile.

Questa dottrina del Maimonide del tempo è calcata da quella di Aristotele, anche se Aristotele indica il tempo anzichè un accidente di un accidente, una qualità di qualità. Quanto il Maimonide dice del tempo a proposito del problema dell'eternità del mondo, è un completamento di quanto dice a proposito della venticinquesima proposizione aristotelica, cioè di Aristotele e della sua scuola, come egli indica esplicitamente.

Dunque per il Maimonide il tempo non è né un'astrazione, né un qualcosa di soggettivo, bensì è una realtà che non sta senza un'altra realtà, cosi come, l'esempio è del Maimonide, la curva che è un accidente di un altro accidente che è la linea e senza linea non vi è curva. Questa visione del tempo serve al Maimonide per due

\footnotetext{
2 «Poichè il tempo è senza dubbio un accidente, ed è, secondo la nostra conoscenza, uno degli accidenti creati, come la nerezza e la bianchezza, tuttavia [esso] non è della stessa specie [degli accidenti] della qualità". Y. KafAH (ed), רבנ משה בן מימון מורה הנבוכים, Jerusalem 1972, p. 190 (d'ora in poi Môreh).

3 «Perchè gli accidenti che esistono nei corpi esistono primieramente come il colore nella vista e sapore. [Essi], in verità si concepiscono nel loro significato primo e si intendono nel loro significato; ma [vi sono] accidenti il cui soggetto sono altri accidenti come lo splendore nella vista e la curva o la o la rotondità nella linea». Ibid.

${ }^{4}$ «[Gli accidenti di accidenti] sono di significato molto difficile, specialmente se si aggiunge a questo che l'accidente [che funge] da soggetto non è stabile nella sua condizione, perchè è una condizione dopo una condizione, allora l'oscurità è maggiore. Ora nel tempo sono riunite le due cose insieme, poichè [esso] è un accidente che è legato al movimento, ed il movimento è un accidente di ciò che è mosso». Ibid.
} 
scopi: assicurare che ciò che è immobile è fuori del tempo o della temporalità, essendo il tempo accidente del moto, e tale è Dio; risolvere il problema del tempo prima della creazione. Prima che vi fosse il mondo il tempo non esisteva; il tempo nacque o fu creato, quando nacque o fu creato il moto, ed il moto nacque con il mondo. Il tempo è pertanto anch'esso una creatura di Dio. Il mondo non fu creato nel tempo, ma con il tempo.

Con questo il Maimonide ha risposto ad una possibile domanda, che nella Guida non si legge, ma che è implicita, e cioè: quanto tempo trascorse prima della creazione dell'universo, o quanto tempo Dio attese prima della creazione? Domanda ormai senza significato, poichè prima della creazione non esisteva alcun tempo.

Chiarito il problema del tempo, possiamo entrare nell'argomento dell'eternità o meno del mondo; su questo tema dell'eternità il Maimonide ci dice che vi sono tre opinioni. La prima è quella dei credenti nella Legge mosaica, i quali sostengono che il mondo non è eterno, ma che esso è stato «creato da Dio dopo ('aharê) il nulla assoluto e totale», e la creazione è il frutto della volontà e del desiderio di Dio ${ }^{5}$. La seconda è la posizione propria di quei filosofi che egli ha conosciuto direttamente o indirettamente, $i$ quali dicono che il comparire delle cose dal nulla è una contraddizione, così come il loro scomparire nel nulla; essi negano pertanto che Dio abbia creato qualcosa dal nulla, «che Dio abbia creato, ciò che c"è, dal nulla», e pur ammettendo che Dio è causa del mondo, questi tali sostengono l'esistenza di una materia eterna, come eterno è Dio. Per questa dottrina, attribuita genericamente ai filosofi, il Maimonide cita esplicitamente soltanto il Timeo di Platone ${ }^{6}$. La terza opinione è quella, dice, di Aristotele e dei suoi seguaci, i quali sostengono che "non esiste una cosa corporea senza [che esista] affatto una materia". Dicono che il cielo non nasce e che non si corrompe, e «la cosa che è stabile non è sottoposta alla nascita e alla corruzione" '? Il motivo di questo, fa capire il Maimonide, è che Dio non può cambiare la sua volontà, come avverrebbe nel caso di un mondo creato.

Dunque, con onestà, il Maimonide che conosce Aristotele diret-

\footnotetext{
${ }^{5}$ Môreh, p. 189.

${ }^{6}$ Moreh, pp. 190-191.

' Môreh, p. 191.
} 
tamente e che ha degli altri filosofi una conoscenza soltanto parzialmente diretta, ha distinto la posizione di Aristotele e dei suoi seguaci da quella degli altri filosofi. In realtà sul tema dell'eternità la posizione di Aristotele è uguale a quella di Platone e degli altri pensatori greci, con la differenza che essa è più articolata. La distinzione che fa il Maimonide ha però uno scopo, e noi lo vedremo più avanti, quello di tentare una conciliazione della posizione di Aristotele con quella della rivelazione mosaica.

Comunque, poste queste tre posizioni, possiamo riassumere la questione in questo modo: la rivelazione ci dice che il mondo è stato creato secondo la volontà di Dio e che perciò esso non è eterno; i filosofi hanno sostenuto che il mondo non è stato creato e che perciò esso è eterno. La non eternità del mondo è dunque una verità di fede in contrasto con la opinione dei filosofi. Rimane il problema teorico, la non eternità del mondo è anche una verità di ragione, nel senso che è essa dimostrabile razionalmente? E la opinione dei filosofi è proprio una verità di ragione? Poichè due verità sullo stesso tema non possono esistere, si tratta di stabilire se vi sono e quali sono le prove valide a favore dell'una o dell'altra tesi in contrasto. Poichè gli aristotelici hanno portati una serie di argomenti in favore dell'eternità del mondo, si tratta di sottoporre questi argomenti ad una critica per valutare il loro valore probante. Se il risultato sarà positivo, nel senso di poter affermare che esiste una dimostrazione razionale valida circa l'eternità del mondo, allora si deve concludere che la Rivelazione mosaica è errata. In caso contrario, se tali argomenti, al vaglio della critica, mostrano di non aver valore, allora il problema è aperto, ed occorre esaminare se hanno valore probante gli argomenti portati in favore della non eternità del mondo. Se hanno tale valore, la non eternità del mondo non è soltanto una verità di fede valida, ma è anche una verità di ragione, in caso contrario il problema della eternità o meno del mondo rimane come verità di fede, ma dal punto di vista razionale esso è insolubile. Quest'ultima posizione, diciamolo subito, non è la posizione sostenuta dal Maimonide, ma è vicina al suo pensiero. Questo è anche lo schema del suo procedimento che qui vogliamo esaminare.

Il Maimonide riporta prima di tutto gli argomenti aristotelici in 
favore della eternità del mondo, questi argomenti sono sette. Il primo argomento (derek ) è fondato sul movimento. Il Maimonide scrive: "Aristotele dice che il movimento non è stato fatto (hiwwâ) e non perirà..." se il movimento fosse ex novo, allora, (poichè) ogni cosa essendo preceduta da un moto che la fa passare all'atto, ne deriverebbe che ogni moto è preceduto da un moto. Il movimento è dunque eterno ed eterno è pure, aggiunge, il tempo. Questo argomento è un riassunto di quanto Aristotele ha scritto nell'ottavo libro della sua Fisica ${ }^{8}$. Il secondo argomento, che è pure tratto dalla stessa opera di Aristotele, è fondato sulla materia prima. Essa, dice, non è stata fatta e non perirà, "poichè se la materia prima fossa stata fatta, allora esisterebbe una materia dalla quale è stata fatta»; dunque, esiste comunque da sempre una materia ${ }^{9}$. Il terzo è fondato sulla materia della sfera celeste. Questa materia, leggiamo nel Maimonide, «non ha alcuna specie di contrario, perchè il moto circolare non ha contrari». Ora, essendo il perire opera di contrari che esistono in ciò che perisce, la materia della sfera celeste non perisce, ma ciò che non perisce non è stato fatto, dunque la materia della sfera celeste non è stata fatta e perciò essa è eterna ${ }^{10}$. Il quarto argomento è fondato sulla possibilità. $\mathrm{Il}$ mondo prima che fosse non era impedito, la sua nascita era o possibile, o necessaria, o impossibile. Se era necessaria il mondo è eterno, se impossibile il mondo non sarebbe, se infine possibile, allora qual'è il substrato o il soggetto di questa possibilità? Bisogna ammettere la esistenza di un substrato della possibilità ${ }^{11}$.

Questo quarto argomento non è di Aristotele, ed il Maimonide lo fa un po' capire, dice però che questo è un argomento molto forte e che alcuni Teologi musulmani del suo tempo hanno cercato di confutarlo dicendo che la possibilità sta nel soggetto e non nell'oggetto dell'azione, ma la loro confutazione, dice il Maimonide, non convince.

Il quinto argomento si fonda sull'impossibilità della creazione da parte di Dio. Esso dice che se Dio avesse prodotto il mondo dopo il

\footnotetext{
${ }^{8}$ Môreh, pp. 193-194.

${ }^{9}$ Môreh, p. 194.

${ }^{10}$ Ibid.

"Ibid.
} 
nulla, allora Dio prima della creazione del mondo era un agente in potenza; e quando Dio creò divenne agente in atto. Questo vuol dire che Dio è passato dalla potenza all'atto. Allora Dio ha avuto bisogno di un agente, il chè è assurdo ${ }^{12}$. La sesta argomentazione si fonda sulla necessità dell'azione di Dio. «Se un agente non è agente in un tempo ed è agente in un altro tempo", allora è perchè vi sono ostacoli ed impedimenti in lui, ma ciò è assurdo ${ }^{13}$. L'ultimo, infine, argomento è fondato sulla perfezione delle opere di Dio. «Le opere di Dio sono molto perfette e non vi è in esse difetto, inutilità e superfluo", se è così "occorre che [il mondo] sia perpetuo poichè è perpetua, come la Sua essenza, la saggezza Sua [di Dio]» ${ }^{14}$. A questi sette argomenti il Maimonide ne aggiunge uno tutto particolare, ricavato anche questo da Aristotele, un argomento fondato sul consenso dei popoli: "Aristotele dice che tutto il mondo crede nella perpetuità del cielo e nella sua stabilità", per questo ha fatto del cielo la dimora di Dio e degli angeli ${ }^{15}$.

Non è inutile, a questo punto, fare un bilancio di questi vari argomenti riportati dal Maimonide. Di questi sette argomenti egli attribuisce i primi quattro ad Aristotele e gli altri tre a quelli che egli indica come i suoi seguaci. Per dei quattro primi argomenti il Maimonide sembra dubitare della loro autenticità, essi in realtà derivano da una interpretazione di al-Farabi; dei tre argomenti del secondo gruppo il Maimonide non ci indica alcun autore. Essi nella sostanza derivano da Avicenna, certamente quello fondato sulla necessità dell'azione di Dio e perciò della immediatezza di essa, e così quello fondato sulla possibilità dell'agire e del non agire di Dio. Il Maimonide ha potuto trarre questi argomenti dalla Metafisica di Avicenna. Più difficile è dire dell'autore dell'ultimo argomento fondato su un certo ottimismo cosmologico. In tutti questi argomenti il Maimonide ha riconosciuto una forza di convincimento, anche se non probante, di due di essi, del quarto in modo particolare, come abbiamo visto, e del quinto del quale dice che esso fa meditare per risolverlo.

\footnotetext{
${ }^{12}$ Môreh, p. 195.

${ }^{13}$ Ibid.

${ }^{14}$ Ibid.

${ }^{15}$ Môreh, pp. 195-196.
} 
Ora di fronte a questi sette argomenti l'atteggiamento del Maimonide è articolato ed interessante. Prima di tutto egli si preoccupa di giustificare Aristotele ed in questo modo infirma il valore dei primi argomenti. Il Maimonide dice che Aristotele non considerava questi argomenti come delle vere prove. È bene citare qui le parole stesse del Maimonide: «La mia intenzione in questo capitolo è di chiarire che Aristotele non possiede [alcuna] prova circa l'eternità del mondo, secondo la sua [stessa] opinione, ed in ciò non sbaglia; vale a dire che egli stesso sa che non vi è prova su ciò. Questi argomenti di dimostrazione e prove, che egli chiama argomenti, sono essi apparenza e tendono [soltanto] verso la conoscenza di più ... Non vi è da credere che Aristotele pensi che questi argomenti sieno come una dimostrazione» ${ }^{16}$.

Detto questo noi dobbiamo notare, per comprendere l'atteggiamento del Maimonide rispetto a questi argomenti, che i primi quattro di essi, quelli di Aristotele, sono fondati sull'analisi del mondo, ma gli argomenti fondati sull'analisi del mondo, dirà più avanti il Maimonide, non servono per il nostro problema; gli altri tre sono invece fondati sull'analisi dell'azione divina. Per questo la confutazione del Maimonide è diversa rispetto ai due gruppi di argomenti. Agli ultimi tre egli dedica tre confutazioni, una per argomento, ai primi quattro invece dedica una confutazione unica, che poi applica brevemente ai singoli argomenti.

Per i primi quattro argomenti la confutazione del Maimonide si fonda su questa convinzione: questi argomenti sono fondati sul mondo qual'è, sono una extrapolazione, per dirla in termini moderni, della situazione del mondo creato e ormai perfetto, ma proprio per questo tali argomenti non valgono per negare la possibilità della creazione e perciò della non eternità del mondo. In una realtà in divenire occorre distinguere, dice il Maimonide, tre stati fondamentali: lo stato di questa realtà prima del suo divenire, il suo stato durante il divenire e lo stato di questa realtà a divenire compiuto. Ciò che noi qui abbiamo chiamato realtà, il Maimonide dice natura. A questo fine egli porta l'esempio del divenire dell'uomo, nel quale bisogna distinguere lo stato di sostanza organica maschile e femminile, lo stato di gestazione e lo stato dell'uomo nato e compiuto.

${ }^{16}$ Môreh, p. 196. 
Ora, egli dice, pretendere di stabilire, attraverso una indagine sulla stato di realtà compiuta come era, prima che così fosse, è impossibile; se un uomo conoscesse soltanto il suo stato di uomo formato non potrebbe mai sapere, da una indagine su se stesso, come egli era prima di nascere ${ }^{17}$.

Qualsiasi argomento può avere una sua logica, ma il limite della sua validità non può superare la barriera dello stato attuale di ciò su cui si è indagato. Gli argomenti di Aristotele, secondo il Maimonide, valgono per il mondo creato, non per il mondo prima della sua creazione, quando il mondo non era. Notiamo, prima della applicazione di questo ragionamento ai singoli argomenti di Aristotele, come il Maimonide presuppone una soluzione di continuità tra i tre stati o tre nature, per cui indagando su una non si può passare a conoscere l'altra. Questa soluzione di continuità tra stati diversi è valido nel passaggio tre il non-essere e l'essere, cioè a proposito della creazione, mentre non è valido per il passaggio dalla potenza all'atta. Ci sembra che il Maimonide abbia intuito la profonda differenza tra i due movimenti, ma il suo pensiero non è molto chiaro su questo punto importante. Comunque egli dice: "Aristotele viene a confutare le nostre parole, ma porta contro di noi un argomento [tratto] dalla natura dell'essere [già] arrivato, perfetto, esistente in atto; [mentre] noi pensiamo che esso dopo l'arrivo non assomigli affatto a ciò che era al tempo della nascita" ${ }^{18}$.

Posto questo fatto della inapplicabilità in linea generale degli argomenti portati da Aristotele e che Aristotele stesso, al dire del Maimonide, non li riteneva probanti, è facile la confutazione degli argomenti presi singolarmente. Che la materia non sia stata fatta è vero, dice il Maimonide, ma questo non vuol dire che essa sia eterna, «Essa non è stata fatta come è stato fatto ciò che è stato fatto da essa, e non perisce come perisce ciò che perisce in essa, ma è stata creata e quando vuole il suo Creatore la ridurrà nel nulla puro ed assoluto» ${ }^{19}$. Che il moto circolare non abbia inizio è vero, ma questo vale soltando dopo che il corpo sferico è stato prodotto. Lo stesso vale per la possibilità, è vero che essa precede ciò che è

\footnotetext{
${ }^{17}$ Môreh, pp. 199-200.

${ }^{18}$ Môreh, p. 200.

${ }^{19}$ Ibid.
} 
stato fatto, ma non precede ciò che deriva dal nulla. Infine che il cielo non abbia contrarietà, è ancora vero, ma noi non diciamo che il cielo sia stato fatto come sono fatte le altre cose una dall'altra, né diciamo che il cielo perisce perchè è composto ${ }^{20}$.

Liberatosi, se così si può dire, di questo primo gruppo di argomenti, il Maimonide esamina ora uno dopo l'altro gli altri tre. Quanto al primo che afferma che la creazione imporrebbe a Dio un passaggio dalla potenza all'atto, questo vale, egli dice, soltanto a proposito «di ciò che è composto di una materia che possiede la possibilità e di una forma» ${ }^{21}$; non vale invece a proposito di Dio il quale non avendo in sé una materia non ha possibilità. Il secondo argomento è quello che afferma la produzione ab aeterno del mondo, salvo a supporre degli impedimenti all'azione divina. La confutazione di questo argomento riconosce il Maimonide è difficile e pertanto la sua confutazione ha bisogno di una sua attenzione.

Il ragionamento del Maimonide è che un conto è la volontà degli uomini ed un altro è la volontà di Dio; la volontà degli uomini è soggetta ad azioni esterne su di essa, non quella di Dio; una ha uno scopo al di fuori di essa, quella di Dio no. Cerchiamo di seguire il più da vicino possibile il complesso ragionamento del Maimonide. Un uomo, dice, può volere costruire una casa, ma può avere motivi vari per non costruirla: mancanza dei mezzi, fatti o avvenimenti che consigliano di non costruirla. «È dunque chiaro che gli avvenimenti cambiano la volontà», ma se la volontà non ubbidisce a motivi esterni ad essa, così non è. "Ciononostante non è necessario, se non vi son ostacoli, che la volontà agisca sempre, perchè non avendo uno scopo al di fuori, non vi è motivo che la spinga ad agire». Aggiunge il Maimonide: «Ma se [una volontà] vuole e non vuole vi è [forse] cambiamento? No, perchè ciò che costituisce la verità della volontà e la sua essenza è che vuole e non vuole». Se si tratta della volontà di un essere materiale, allora essa, avendo un fine esterno, dipende da ostacoli e da avvenimenti, ma la volontà di Colui che è separato (dalla materia) non è per qualche causa che non attua, $e$ il fatto che ora vuole una cosa e domani un'altra non cambia la sua essenza. Il Maimonide conclude dicendo che «la

\footnotetext{
20 Ibid.

21 Môreh, p. 202.
} 
nostra volontà e la volontà dell'Essere separato sono due volontà in senso omonimo (b̆ěsitûff), ma che tra le due volontà non vi è somiglianza (dimîon)» ${ }^{22}$.

A proposito di questa lunga confutazione del Maimonide e specialmente dell'ultima affermazione occorre fare una doppia considerazione, che ci collega con il problema degli attributi di Dio. Dire che la volontà umana e la volontà divina sono due volontà diverse è ciò che il Maimonide ha dimostrato con argomenti convincenti, ma affermare che tra le due non vi è somiglianza e che in comune hanno soltanto il nome, non sembra esatto, anche secondo il Maimonide, salvo a dare un significato diverso ai due termini usati di sitûf e di dimîôn, nome in comune e somiglianza. Egli ci ha detto che la natura della volontà è volere e non volere, ora questa natura o questo significato vale sia per la volontà dell'uomo che per la volontà di Dio. Il termine volontà applicato all'uomo e a Dio è qualcosa di più che un nome comune o omonimo, se potessimo usare un'espressione della teologia cristiana diremmo che siamo di fronte ad un termine analogo. Inoltre il Maimonide ci ha detto che Dio vuole e non vuole, che Egli agisce con intenzione, anche se il Suo agire ed il Suo volere è diverso da quello degli uomini, ci sembra che qui il Maimonide abbia attribuito a Dio qualcosa che non è soltanto negativo.

Il terzo argomento è quello della perfezione e perciò della eternità delle opere di Dio. A questo argomento il Maimonide oppone che il far ricorso alla saggezza di Dio è un argomento debole perchè noi, della saggezza di Dio, nulla sappiamo e non possiamo, proprio per questo, dire ad esempio, perchè il numero delle sfere celesti, è così e non altrimenti ${ }^{23}$. Infine all'argomento aggiuntivo che fa appello all'opinione comune dei popoli circa la perpetuità del cielo e della sua stabilità, il Maimonide afferma che esso non è un argomento razionale e perciò non è probante ${ }^{24}$.

Con queste sue ultime argomentazioni il Maimonide ha dimostrato che storicamente nessuno ha portato una prova razionale valida a mostrare che il mondo è eterno. Da questa conclusione

\footnotetext{
${ }^{22}$ Môreh, p. 202-203.

${ }^{23}$ Môreh, pp. 203.

${ }^{24}$ Ibid.
} 
storica il Maimonide crede di poter trarre anche una conclusione teorica e cioè che non è dimostrabile razionalmente che il mondo sia eterno, e da questa conclusione teorica il Maimonide ne deduce, come sua conseguenza, che dal punto di vista della ragione umana non è impossibile, così le sue parole al termine di tutto il dibattito, che il mondo non sia eterno; non è impossibile si intende sotto l'aspetto della ragione. Il Maimonide, dobbiamo ricordarlo, aveva precedentemente confutato le varie argomentazioni portate dai teologi musulmani, i mutakallemin, in favore della prova razionale che il mondo è stato creato, e per conseguenza, secondo il Maimonide, che il mondo non è eterno. Anche qui da una conclusione storica, non esistono storicamente prove atte a dimostrare razionalmente che il mondo è stato creato e perciò non eterno, ne aveva tratto una conclusione teorica: la creazione del mondo e con essa la sua non eternità non è razionalmente dimostrabile.

Il problema dunque, sotto l'aspetto della ragione, è indeciso ed insolubile. Questa sembra fin qui la conclusione e la posizione del Maimonide di fronte al problema se il mondo sia eterno eppure non eterno. In realtà così non è. La Guida è stata dal Maimonide scritta proprio per guidare gli incerti nel rapporto tra le affermazioni letterali della Sacra Scrittura e la ragione umana, con i suoi argomenti e le sue conquiste. Questa sua caratteristica è presente anche a proposito di questo tema ed è a questo punto che il Maimonide inserisce una sua particolare argomentazione che precisa la sua posizione.

Prima di esaminare questa parte importante, la più importante secondo il Munk, in tutta la discussione contro i peripatetici, è bene fare un breve riassunto delle varie posizioni che i pensatori ed $\mathrm{i}$ teologi hanno assunto nella storia; questo varrà a collocare meglio la posizione del Maimonide sul tema della eternità o meno del mondo.

Se le opinioni sulla eternità o meno del mondo non sono, e non possono essere, che due: il mondo è eterno o il mondo eterno non è, le opinioni invece circa la dimostrabilità o meno dell'eternità del mondo o della sua non eternità sono diverse; il problema cioè delle prove razionali atte a risolvere, secondo ragione, questo tema, presenta soluzioni più articolate. Il Maimonide stesso, sia pure in modo 
non sistematico, ma inframezzando le notizie lungo la sua esposizione, ci dà un quadro di questa articolazione di posizioni. Egli ci dice infatti che vi è la posizione di coloro che egli chiama i Seguaci di Aristotele, che sono in realtà seguaci di al-Farabi e di Avicenna, $i$ quali sostengono non soltanto che il mondo è eterno, ma sostengono che la sua eternità è dimostrabile razionalmente, e portano essi stessi le prove di ragione. Vi è la posizione di Aristotele il quale sostiene che il mondo è eterno, che porta anche argomenti in favore di questa eternità, ma questi argomenti, per confessione dello stesso Aristotele, non sono delle vere prove razionali. Su quest'ultimo punto, cioè sul valore degli argomenti di Aristotele, vi è inoltre la posizione di al-Farabi, il quale afferma che Aristotele ha dimostrato, con prove aventi valore, razionalmente che il mondo è eterno. Vi è poi la posizione dei teologi musulmani, i mutakallemin, i quali non soltanto sostengono che il mondo non è eterno, ma di questa sua non eternità portano delle prove, prove che vogliono dimostrare razionalmente che il mondo è stato creato, ed in conseguenza che esso non è eterno; queste prove dei Teologi musulmani non valgono, secondo il pensiero del Maimonide, a dimostrare che il mondo è stato creato e per conseguenza non valgono neppure a dimostrare che esso non è eterno. Infine vi è la posizione di Galeno il quale sostiene che è futile ed inutile discutere se il mondo sia eterno oppure no ${ }^{25}$.

È di fronte a questo quadro, con le relative interpretazioni, che si colloca la posizione del nostro Autore; essa acquista particolare rilievo proprio calata in queste articolate posizioni. La sua posizione può essere così anticipata e riassunta: che il mondo sia eterno non è stato dimostrato, come non è stato dimostrato che esso non sia eterno, gli argomenti storicamente portati in favore e dell'una e dell'altra posizione non hanno valore; non è vero però che tale questione sia futile ed inutile. Il problema sotto l'aspetto razionale è insolubile. Il mondo però non è eterno, esso non è eterno perchè il mondo è stato creato, questa è una verità di fede e non di ragione; tuttavia, esiste una argomentazione razionale in favore della opinione della non eternità del mondo, questa argomentazione, che finora nessuno ha portato, pur non avendo valore probante, fa pendere la

\footnotetext{
${ }^{25}$ Môreh, p. 197.
} 
nostra ragione più verso la opinione che il mondo non è eterno che verso la opinione che il mondo è eterno.

Poichè il modo di argomentare che il Maimonide fa a questo proposito è importante e di non sempre facile comprensione occorre che noi, anche a questo proposito, procediamo con la maggior aderenza possibile alle sue parole. Egli inizia il suo argomento con due precisazioni metodologiche. La prima è che egli vuole portare un argomento vicino a una dimostrazione razionale, letteralmente il Maimonide dice: «Il mio scopo, in questo capitolo, è di spiegare a te, con prove vicine ad essere una dimostrazione» ${ }^{26}$. Non intende dunque portare un argomento probante, che non esiste, come abbiamo visto, quanto piuttosto un argomento convincente, nel senso che esso sia capace di spostare l'equilibrio dell'incertezza razionale, e faccia razionalmente piegare dalla parte della non eternità e perciò dalla creazione del mondo. La seconda precisazione è una raccomandazione, il Maimonide avverte che la sua argomentazione è simile alla argomentazione portata dai Mutakallemin indicata come argomentazione della determinazione (yihûud), la sua argomentazione è simile, ma non deve essere confusa con essa; se identico è lo scopo, diverso è il procedimento ed il fondamento.

Questa argomentazione parte dalla considerazione del modo di essere dell'universo, e ad esso egli applica un principio di ordine generale. Questo principio, che da tutti deve essere accolto, dice: "Ogni [volta che una] materia è comune a cose diverse, in qualunque modo sieno diverse, porta necessariamente ad un'altra causa, fuori della materia comune, la quale [causa] è essa che determina che una parte sia di un aspetto particolare ed una parte di un altro aspetto, oppure a cause diverse quanto sono le cose [diverse]» ${ }^{27}$. Poichè tutta la argomentazione del Maimonide, per sua stessa confessione, appoggia su questo principio è bene soffermarci brevemente su di esso. Questo principio stabilisce la insufficienza causale della materia rispetto alla diversità delle cose, o degli aspetti delle cose. Esso chiama in causa i sostenitori della materia prima come causa universale che non può essere anche causa particolare. Che esista una materia causa delle cose il Maimonide non nega, è la causa detta

\footnotetext{
${ }^{26}$ Môreh, p. 204.

${ }^{27}$ Ibid.
} 
materiale; ma fa notare che essa, da sola, non può giustificare la diversità delle cose. Occorre, in questo caso, ammettere una delle due: o la esistenza di una causa della diversità o la esistenza di diverse cause, quante sono le cose diverse radicate, se così si può dire, in una sola materia.

Stabilito questo principio, che secondo il Maimonide è accettabile sia dai sostenitori della eternità del mondo, che dai sostenitori della sua non eternità, egli procede alla sua applicazione in un modo piuttosto originale. Procederò ora, egli dice, a discutere l'opinione di Aristotele, "con la forma della domanda e della risposta». Procede cioè in modo dialettico, con una serie di obbiezioni e di risposte ad ipotetiche contro obbiezioni.

In parole più semplici, l'argomento del Maimonide si svolge sotto forma di due indagini, una sul mondo terrestre ed un'altra sul mondo celeste, mondi che, nella visione cosmologica aristotelica, sono sostanzialmente diversi. La prima indagine inizia con questa domanda: poichè esiste una sola materia terrestre per tutte lo cose fisiche, qual'è la causa della differenza tra le varie specie? E qual è la causa della differenza dei vari individui in ciascuna specie? Cioè, se la materia è unica, come si spiega la molteplicità e la diversità delle specie, e degli individui nell'ambito di una specie? Queste le parole del Maimonide: «Domandiamo ad Aristotele e diciamo a lui, poichè ci è stato dimostrato che la materia di tutte le cose [che stanno] sotto la sfera della luna è una materia unica comune a tutte [le cose], se così è, qual'è la causa della diversità di tutte le specie che si trovano qui, e qual'è la causa degli individui di ogni specie?» ${ }^{28}$.

Se Aristotele, aggiunge il Maimonide, risponde che la materia ha ricevuto dapprima quattro forme, da cui i quattro elementi fondamentali, e questi, per il movimento della sfera celeste, si mescolano e si dividono, essi per la loro mescolanza (mëzîgâ), vengono disposti a ricevere altre forme diverse, e così continuamente; le forme poi delle specie si determinano ulteriormente e ricevono altre forme da cui nascono gli individui di ciascuna specie. Se Aristotele così risponde, dice il Maimonide, noi diciamo che ciò è vero, ma allora domandiamo se è la mescolanza che dispone la materia prima, o comune, a ricevere le varie forme, che cosa è o qual'è la causa per

\footnotetext{
${ }^{28}$ Môreh, p. 205.
} 
cui una parte della materia riceve ad esempio la forma del fuoco ed un'altra parte la forma della terra e così via? A questa domanda, Aristotele non ha che una risposta: ciò che dispone una parte della materia a ricevere una determinata forma ed un'altra parte a ricevere un'altra forma è la diversità dei luoghi ove stanno le varie parti della materia, "poichè sono essi [i luoghi] che necessariamente, a questa unica materia, [danno] disposizioni diverse». Ammettiamo, dice qui il Maimonide, che sia così, poichè è difficile trovare un'altra spiegazione ${ }^{29}$.

Osserviamo ora la materia dei cieli, Aristotele ci dice che essa è diversa da quella sublunare e che nei cieli vi sono altre forme e se usiamo la parola corpo per indicare le cose celesti, questa parola ha per queste cose un altro significato. "Tutto questo è già stato dimostrato", dice il Maimonide. Ma viene ora, fa notare, il punto centrale di tutto il suo argomento: "Ascolta ciò che ti dico su questo argomento, in questa mia esposizione» ${ }^{30}$. Poichè è dalla diversità delle azioni che noi deduciamo la diversità delle forme $e$ poichè tutti i moti naturali delle cose terrestri sono retti, ne deduciamo che vi è una sola materia e che vi sono diverse forme perchè questi moti si diversificano tra di loro; così poichè il moto della sfera è un moto circolare diciamo che vi è una sola materia celeste, e poichè i moti celesti, pur essendo tutti circolari, si diversificano, allora diciamo che vi sono diverse forme celesti. Ora se le varie sfere celesti hanno una sola materia comune ed hanno forme diverse e se la materia comune di ciascuna sfera è il soggetto (o il substratum, nôśs $e^{\jmath}$ ) di una determinata (měyuhedet ) forma, diversa una dall'altra, chi è colui che diversifica (mĕyuhad ) i vari soggetti e li prepara a ricevere le diverse forme? ${ }^{31}$.

A questa domanda, Aristotele con la sua grande acutezza, dice il Maimonide, cercò di dare una risposta che non lo spingesse a ricercare la causa fuori del mondo celeste, egli tentò di spiegare, ad esempio, il perchè della direzione determinata del moto delle sfere, il perchè delle varie velocità del loro moto e perchè i vari pianeti hanno più sfere, mentre molte stelle fisse sono in una sola sfera.

\footnotetext{
${ }^{29}$ Ibid.

${ }^{30}$ Ibid.

${ }^{31}$ Môreh, p. 206.
} 
Egli cercò di dare una risposta a tutti questi interrogativi con motivazioni logiche, che mostrassero la esistenza di un legame di necessità tra tutto ciò che avviene e c'è nelle sfere del cielo, ma il suo sforzo è stato inutile. Aristotele, dice il Maimonide, non è riuscito: «non è riuscito e non riuscirà in eterno", nel senso che nessuno vi riuscirà mai ${ }^{32}$.

Per il Maimonide, la causa della diversificazione delle varie parti della materia celeste preparate a ricevere forme diverse, non è altro che Dio. Letteralmente egli dice: «Vi è da porre, oltre la sfera [celeste] altra cosa da attribuirgli questa diversificazione [o determinazione, yihhûd], se non Dio, sia Egli glorificato ed esaltato?» ${ }^{33}$. La chiave del problema è tutta qui. Se per il mondo fisico, quello sublunare, Aristotele ha potuto darci una dimostrazione convincente della esistenza di un legame logico, e perciò di necessità tra tutte le cose e la loro diversificazione o determinazione o particolarizzazione, ricorrendo al mondo celeste come causa, ciò, per lo stesso mondo celeste non gli è stato possibile, e questo perchè il legame logico di necessità non esiste. II legame necessario ha un suo limite, esso non riesce a dare una risposta, quella ultima, del perchè della diversificazione delle singole sfere e delle diverse loro caratteristiche. La vera risposta è che il legame di necessità non c'è, o esso non è in toto, questo legame se si vuole spiega molto, ma certo non spiega tutto. Merita notare, a questo proposito, questa precisa affermazione del Maimonide: «È stato possibile dire che vi è un legame di necessità fra i moti e fra le forze delle sfere, ma in tutto ciò che richiama l'oggetto della sfera [celeste] non è stata data alcuna causa chiara di essa e non vi è cosa [alcuna] che sia in base ad un ordine, così che sia possibile affermare che vi è, in essa, una necessità» ${ }^{34}$.

È vero, conclude il Maimonide, che per spiegare le varie particolarizzazioni delle sfere Aristotele è ricorso alla teoria che ogni sfera ha una sua intelligenza, ma ciò non risolve il problema. La soluzione del problema è la esistenza di Dio fuori dell'universo, il quale ha voluto che fosse così come è. Il Maimonide aggiunge altri fatti nel campo del mondo celeste che sono inspiegabili con la teoria della

\footnotetext{
${ }^{32}$ Ibid.

${ }^{33}$ Ibid.

${ }^{34}$ Ibid.
} 
necessità. Questi fatti sono le due materie esistenti e le due forme fondamentali celesti ed il loro collegamento, il fatto dell'irregolare raggruppamento delle stelle nella ottava sfera, il fatto che una parte della sfera tocca ad un astro ed un'altra parte ad un altro astro. In altre parole esistono nel cielo varietà di movimenti e di astri fissati nella sfera e varietà di collocazioni, le quali altra spiegazione non hanno che la volontà di Dio che così ha voluto che fosse.

A questo punto il discorso, ormai troppo lungo, impone un richiamo ai punti principali. Il Maimonide voleva convincere che il mondo non è eterno, per tale convinzione egli ha dimostrato che il mondo non è il frutto di una azione necessitata di Dio, bensì di una azione voluta. L'azione necessitata non può non essere e non può essere che in un determinato modo. Ma se il mondo fosse il frutto di una azione necessitata di Dio, esso sarebbe logico in ogni sua parte, ordinato nel suo interno con vincoli di necessità, invece una indagine approfondita di esso e del suo meccanismo, dimostra che se per quanto riguarda il mondo sub-lunare tali vincoli di necessità ed ordine logico esistono, per quanto riguarda il mondo celeste così non è. Qui l'azione divina non può dirsi frutto di necessità, ed allora bisogna concludere che essa è frutto di volontà. Il nostro Autore ha voluto dimostrare che il mondo è sí effetto di Dio, sua causa, ma non è un effetto necessitato. Il rapporto Dio e mondo non è un rapporto di necessità, il mondo non è uscito da Dio come un contenuto da un contenente, uscito come era e non poteva essere diversamente.

Proprio perchè il mondo celeste dimostra una certa illogicità e inspiegabilità, la sua causa va cercata fuori di una analisi soltando deduttiva. La diversificazione della materia del cielo, la quale diversificazione spiega perchè vi sono forme diverse in una comune materia, e che non può essere giustificata, come per il mondo terrestre, con la teoria dei luoghi, spinge è indicare Dio come causa volontaria del modo di essere del cielo. Dobbiamo notare come lo sforzo del Maimonide non è di dimostrare l'esistenza di Dio, problema già risolto, ma la esistenza di Dio dotato di volontà, creatore del mondo. Dunque questo lungo ed ancora non terminato argomento, e questo è il motivo per cui noi lo seguiamo da vicino, vuol dimostrare la esistenza di un Dio trascendente all'universo, un 
Dio persona, nel senso di dotato di libera volontà, che ha creato il mondo come ha voluto crearlo, non come ha dovuto crearlo.

Per questo il Maimonide afferma: "Noi diciamo che cè un Determinante che ha determinato ogni sfera, [che ha cioè determinato] la sua direzione, movimento e velocità, ma noi non conosciamo il modo della sua saggezza nel creare questo [mondo] cosìn ${ }^{35}$. È, affrontando direttamente la questione dell'eternità del mondo, dice che i sostenitori della eternità, che egli identifica con i sostenitori della necessità del mondo, sono costretti a lasciare inspiegati molti problemi essendo oscura la loro soluzione, ed aggiunge, rivolgendosi al suo allievo: "Sappi che secondo la nostra opinione, di noi che crediamo nella novità del mondo, tutto è facile, ed è secondo i nostri principi» ${ }^{36}$. La necessità, secondo il Maimonide, non riesce a spiegare la diversificazione, o la determinazione, o la individualizzazione.

Notiamo le espressioni che il Maimonide usa a questo riguardo, egli non parla esplicitamente qui di libera volontà di Dio, ma dice che bisogna porre un «Determinante che determina (měyahed yihhî́ e parla di progettazione di chi progetta (kavanat měkawwen)». Le due espressioni si completano a vicenda, «Determinante che determina» vuol dire "Diversificante che diversifica» ed anche «Individualizzante che individualizza». «Progetto di chi progetta» vuol dire anche «disegno di uno che tale disegno concepisce», o «intenzione di uno che ha l'intenzione». Il Munk traduce l'espressione «progetto di chi progetta» con 'disegno di un essere che agisce con intenzione', egli svolge l'espressione sintetica del Maimonide per rendere più chiaro il pensiero. Comunque il Maimonide ripete varie volte il suo concetto, come unica spiegazione per giustificare i diversi modi di essere delle sfere e delle cose celesti.

Con questa prima e fondamentale parte della sua argomentazione, il Maimonide ha confutato la teoria della necessità mostrando come sia il mondo stesso, quello celeste specialmente, che spinge a postulare la esistenza di un Essere che in possesso di un disegno, di un progetto e di un'idea, ha avuto l'intenzione di realizzarla e l'ha intenzionalmente realizzata. Stabilito questo il Maimonide deve ora

\footnotetext{
${ }^{35}$ Môreh, p. 207.
}

${ }^{36}$ Ibid. 
risolvere l'altra parte, e cioè se è conciliabile l'esistenza di questo Essere che agisce intenzionalmente con la teoria della eternità del mondo. A dire il vero la soluzione è già implicita da ciò che il Maimonide ci ha detto fin qui, egli però ritorna sull'argomento per precisare meglio il suo pensiero, questo perchè, come egli dice, vi sono stati pensatori che pur ammettendo l'esistenza di un Essere che agisce con intenzione, e con intenzione volontaria, sostengono ugualmente che il mondo è eterno.

Il motivo della seconda parte dell'argomentazione del Maimonide è data da questo fatto che riportiamo con le parole del suo autore: «Poichè tra i filosofi posteriori credenti nella eternità del mondo, vi è chi dice che Dio, sia Egli esaltato, è il costruttore del mondo e Colui che ha deciso l'essere di esso e che è per la Sua intenzione e determinazione che è così; tuttavia non è possibile che il mondo sia in un tempo e non in un [altro] tempo, ma come fu, sempre sarà. [Questi] dicono che non è comprensibile che un agente che faccia una cosa sia prima della sua azione nel tempon ${ }^{37}$. Queste parole del Maimonide ci dicono che al suo tempo o vicino al suo tempo, vi erano dei credenti in Dio come causa del mondo, ma sostenitori della eternità del mondo e che l'argomentazione di questi tali, cioè il motivo della loro credenza nell'eternità del mondo, era la impossibilità della esistenza di Dio staccato, nel tempo, dalla sua azione. Sostenere questo, secondo questi tali, era ammettere una certa privazione in Dio.

Chi erano questi filosofi non è difficile da scoprire, poichè anche al-Gazzali li aveva combattuti e cioè al-Farabi e più tardi, Averroè, dunque certi filosofi musulmani? Questi pensatori sostenevano, a modo loro, una creazione immediata, non potendo essi ammettere una distinzione tra la potenza di Dio e la Sua azione, sostenere tale distinzione era per essi ammettere un cambiamento in Dio e ammettere l'essere Dio in potenza. Questa posizione è considerata dal Maimonide quasi una obbiezione alla sua tesi della non eternità, quasi a dire: se tu poni il mondo non eterno tu poni una imperfezione o una privazione in Dio.

Diciamo qui che il problema come obbiezione alla tesi del Maimonide è ben impostato, il Maimonide deve ora superare lo scoglio

\footnotetext{
${ }^{37}$ Môreh, p. 211.
} 
del come sia conciliabile l'apparire all'essere del mondo con la perfezione ed immutabilità di Dio. Posto il problema in questi termini ci si attende che la seconda parte della argomentazione del Maimonide affronti decisamente questo ostacolo e dimostri la coesistenza della non eternità del mondo con la perfezione di Dio. Il Maimonide invece procede altrimenti, egli ci fa un discorso molto diffuso, non facile, senza molto vigore e originalità. Egli fonda questa sua risposta alla obbiezione su due convinzioni: la prima è che, secondo il Maimonide, questa nuova teoria si differenzia dalla precedente più per l'apparenza che nella realtà, cioè che anche questi filosofi sostengono un legame di necessità tra il mondo e Dio: "È dunque chiaro a te, che esamini il mio Trattato, che questi tali hanno cambiato la parola "necessità", ma hanno lasciato sussistere il suo significato" ${ }^{38}$. La seconda è che questi tali non hanno afferrato con esattezza il concetto di progetto o intenzione che noi, dice il Maimonide, abbiamo spiegato e cioè che «il mondo è stato fatto con la progettazione di un progetto".

Queste convinzioni in fondo vogliono dire che ciò che è stato detto contro la tesi della necessità della produzione del mondo, vale anche per questa teoria apparentemente nuova. Qui il Maimonide commette un errore storico, quello di identificare sostanzialmente la teoria della creazione immediata o ab aeterno con la teoria della necessità e della emanazione, e la sua risposta non punta sulla coesistenza della creazione ex novo del mondo e della perfezione di Dio, quanto piuttosto su una ulteriore analisi delle caratteristiche del mondo celeste.

Si ha l'impressione che il Maimonide a questo punto sia stato preso da uno scrupolo, quello di non aver spiegato a sufficienza la sua teoria della determinazione o della particolarizzazione. Egli infatti introduce la sua risposta con queste parole: «Quando tu comprenderai bene il significato [della mia teoria] e conoscerai che non è vero che il mondo è necessariamente dalla esistenza di Dio, [come] l'effetto è necessario rispetto alla sua causa, ma che il mondo è [derivato] dall'azione di Dio o dalla sua determinazione» ${ }^{39}$. Notiamo qui la contrapposizione che il Maimonide fa tra un derivare

\footnotetext{
${ }^{38}$ Ibid.
}

${ }^{39}$ Ibid. 
per necessità ed un derivare per una azione o determinazione o particolarizzazione; e come un poco più avanti egli opponga la necessità all'azione con determinazione e volontà. Ove nel caso della necessità si presuppone una non-azione, nel secondo si presuppone una azione cioè una volontà. Non-azione e azione nell'ambito di dio. Nel primo caso, così è perchè così fu, nel secondo caso, così è perchè così non fu. L'azione necessaria è per il Maimonide in fondo una non-azione, presupponendo l'azione un ex novo. Visione astratta di Dio, nel primo caso, visione personalistica di Dio nel secondo caso.

Lo sforzo del Maimonide per superare la obbiezione è una nuova analisi del creato, dei cieli in particolare, per dimostrare che vi fu una azione, una particolare azione voluta da Dio. Il Maimonide dimostra che la teoria cosmologica aristotelica e neoplatonica delle sfere celesti derivate una dall'altra a cominciare dalla prima, nega la esistenza successiva delle sfere anche se il linguaggio usato può ingannare, nello stesso modo che le qualità secondarie non sono esistenti successivamente alle qualità primarie, anche se il linguaggio sembra dire diversamente. Inoltre, dice il Maimonide, la teoria di Aristotele parte dal principio che da una cosa semplice non può derivare che un'altra cosa semplice. Diciamo anche qui di passaggio, che questo è un principio implicito della dottrina neo-platonica $\mathrm{e}$ non di quella aristotelica. Dunque Dio essendo uno e semplice non ha potuto produrre che una cosa una e semplice, cioè la prima intelligenza ${ }^{40}$.

Non occorre seguire qui l'analisi minuta della cosmologia aristotelico-neoplatonica che il Maimonide fa per seguire le linee maestre della sua argomentazione, basta per noi cogliere gli elementi più essenziali. Il Maimonide fissa i principi cosmologici e metafisici aristotelici-neoplatonici, con una certa confusione degli uni con gli altri, come abbiamo già notato, per dirci che se si ammette il legame di necessità della produzione dei vari esseri, se si ammette il principio che dall'uno non nasce che un uno, non si riesce a giustificare la complessa e varia motivazione del modo di essere delle cose celesti e ciò anche se si accetta in via di ipotesi, «in stima e opinione", che più ci si allontana dalla Intelligenza prima più si

\footnotetext{
${ }^{40}$ Môreh, p. 212.
} 
moltiplicano le cose prodotte. Questo perchè con i principi aristotelici-neoplatonici non si spiega, ad esempio, la molteplicità intrinseca nelle varie sfere, non si spiega la diversità della materia celeste, né la diversità dei movimenti, e neppure si spiega la esistenza della materia del mondo sublunare che è totalmente diversa da quella celeste.

Se invece si tiene presente il principio che «ogni agente che agisce con progetto e volontà, non secondo natura fa opere diverse e molteplici", e se si ammette che tutto ciò che è stato prodotto, lo è stato con "progettazione che ha progettato" ed è stato fatto e determinato secondo che ha esigito la divina saggezza che non erra, cadono tutti gli interrogativi. Interrogativi che invece sussistono se si prende la via della necessità in luogo di quella di «una volontà volentes ${ }^{41}$.

A questa, che possiamo chiamare seconda serie di argomentazioni, il Maimonide aggiunge ancora una considerazione che è quasi un rovesciamento dell'argomento della perfezione di Dio. Dice egli infatti che se si ammette la teoria della necessità, affermando, come conseguenza di essa, che nulla avviene di nuovo, perchè ciò che è esso già fu, allora si toglie a Dio ogni possibilità di una, anche piccola, modifica del creato, si nega la possibilità a Dio anche di allungare un ala di mosca o il restringimento di un piede d'insetto. Dunque è negando la possibilità del nuovo, che si toglie potenza $\mathrm{e}$ perfezione a Dio e non viceversa ${ }^{42}$.

La conclusione fondamentale di tutto questo lungo argomentare del Maimonide circa la eternità o meno del mondo, argomentare che ha ancora, lo vedremo una sua appendice, la conclusione fondamentale è che tra due opinioni contrarie, come ha detto Alessandro di Afrodisia, bisogna accogliere quella che pone meno dubbi, il chè vuol dire che tra le due opinioni della eternità e della novità del mondo bisogna razionalmente accettare quella della novità che delle due ci crea meno dubbi ${ }^{43}$.

Noi potremmo fermarci qui sul tema del mondo, ma prima di trarre la conclusione finale è bene considerare ancora brevemente ciò che abbiamo chiamato la appendice al suo ragionamento.

\footnotetext{
${ }^{41}$ Môreh, p. 213.

${ }^{42}$ Môreh, p. 214.

${ }^{43}$ Môreh, p. 215.
} 
Questa appendice alla questione della eternità o novità del mondo con la quale il Maimonide chiude, nella sua Guida, l'ampia argomentazione è in realtà una duplice appendice, poichè una prima parte di essa tocca il rapporto tra prova razionale e verità di fede, ed è una giustificazione della sua presa di posizione e della importanza che il tema della eternità o meno del mondo ha rispetto al complesso della sua dottrina, una seconda parte invece riguarda un problema di carattere esegetico rispetto alla affermazione di un dottore del periodo tannaitico.

Circa la prima parte il Maimonide, al termine di questa trattazione, sente il dovere di dichiarare che egli ha preso posizione contro la eternità del mondo non tanto perchè tale sia la posizione della Scrittura, dato che affermazioni bibliche su questo tema possono anche essere interpretate variamente e seconda se ad esse si applica una esegesi letterale o una esegesi approfondita, ma per il motivo che non esiste una dimostrazione razionale che il mondo è eterno e perchè se si accetta questa tesi o teoria si scalza alla radice la fede religiosa mosaica e si arriva a difficoltà insuperabili. Quanto alle affermazioni fatte da R. Eliezer, autore di un gruppo di Capitoli, o Baraytôt $\underline{t}$ che sembrano affermare la eternità del mondo, con espressioni piuttosto poetiche, il Maimonide non si preoccupa molto, accontentandosi di notare che R. Eliezer sostiene la esistenza di due materie quella del mondo sublunare e quella del mondo celeste ${ }^{44}$.

${ }^{44}$ Môreh, pp. 221-222. 


\section{RESUMEN}

El autor expone la posición de Maimónides en relación con el problema de la eternidad o de la no eternidad del mundo. Posición original que influyó sobre la que más tarde tuvo Tomás de Aquino sobre el mismo problema: la imposibilidad de ofrecer una demostración racional en favor tanto de la eternidad como de la no eternidad. Con la diferencia de que Maimónides aporta algunas consideraciones que, sin valor de prueba, inclinan la mente humana hacia la idea de la no eternidad del mundo.

\section{SUMMARY}

The author explains the position of Maimonides regarding the problem of the eternity or non-eternity of the world. It's an original position which later exerted an influence on Thomas Aquinas' assertions about the same problem, namely the impossibility to provide a reasonable proof supporting either idea of the eternity or non-eternity. Maimonides differs in giving some considerations which, having no value as a proof, incline the human mind to the idea of the non-eternity of the world. 